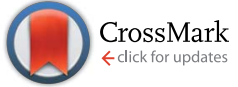

Cite this: J. Mater. Chem. C, 2015, 3, 2204

Received 4th December 2014

Accepted 16th January 2015

DOI: $10.1039 / c 4 t c 02788 d$

www.rsc.org/MaterialsC

\section{Smart polymer inverse-opal photonic crystal films by melt-shear organization for hybrid core-shell architectures $\uparrow$}

\begin{abstract}
C. G. Schäfer, ${ }^{a}$ T. Winter, ${ }^{a}$ S. Heidt, ${ }^{a}$ C. Dietz, ${ }^{\text {b }}$ T. Ding, ${ }^{\text {c }}$ J. J. Baumberg ${ }^{c}$ and M. Gallei ${ }^{\star a}$
A feasible strategy to achieve large-area mechano-, thermo- and solvatochromic hybrid opal (OPC) and inverse opal photonic crystal (IOPC) films based on polymer hydrogels is described. Silica core particles featuring surface-anchored stimuli-responsive polymers are prepared and advantageously used for the melt-shear organization technique. By this approach hybrid OPC films with adjustable periodicities for photonic applications can be prepared. The large-area OPC films can be furthermore converted into IOPC structures simply by etching the silica particles while maintaining the excellent order of the entire opal film. This herein developed new process seems to be universal and is successfully applied to two thermo-responsive polymers, poly( $N$-isopropylacrylamide) (PNIPAM) and poly(diethylene glycol methylether methacrylate) (PDEGMEMA) as particle shell materials. Besides the remarkable mechanical robustness of the hybrid OPC and IOPC films, optical properties upon changes of temperature, mechanical stress and different solvents as external triggers are successfully confirmed. The herein described novel strategy for the preparation of inorganic/organic OPC and IOPC polymer films is feasible for a wide range of applications in fields of sensing and photonic band gap materials.
\end{abstract}

\section{Introduction}

Photonic band gap (PBG) materials have attracted enormous attention due to their wide range of potential applications within the fields of optical (bio)sensing platforms, catalysis, display technologies, actuation systems and security materials..$^{1-7}$ Such PBG materials feature the possibility to modulate the propagation of electromagnetic waves due to their periodic structure forming so-called stop bands. Moreover, there is intensive progress in the field of responsive colloidal crystals, i.e., where the optical properties can be switched by using external triggers such as a change of solvent, temperature, ionic strength, light, electric or magnetic fields or mechanical stress. ${ }^{8-10}$ The first example of a thermo-responsive opal photonic crystal (OPC) structure was reported by Weissman et al. using poly $(\mathrm{N}$-isopropylacrylamide)-based (PNIPAM) particles almost two decades ago. ${ }^{11}$ There are many stimuli-responsive polymer systems which

${ }^{a}$ Ernst-Berl Institut für Technische und Makromolekulare Chemie, Technische Universität Darmstadt, Alarich-Weiss-Str. 4, D-64287 Darmstadt, Germany. E-mail: m.gallei@mc.tu-darmstadt.de

${ }^{b}$ Center of Smart Interfaces and Department of Materials Sciences, Technische Universität Darmstadt, Alarich-Weiss-Str. 10, 64287 Darmstadt, Germany

${ }^{c}$ NanoPhotonics Centre, Cavendish Laboratory, University of Cambridge, Cambridge CB3 OHE, UK

$\dagger$ Electronic supplementary information (ESI) available: TEM images, DLS measurements, table of average particle sizes and standard deviations, DSC measurements and additional UV-Vis reflection spectra. See DOI: $10.1039 / \mathrm{c} 4 \mathrm{tc} 02788 \mathrm{~d}$ have been used for switching the optical properties of (composite) OPC films. Excellent and recent reviews within that field are given by e.g. Ge et al., ${ }^{8}$ Lee et al. ${ }^{12}$ and Lopez et al. ${ }^{4}$ Especially organic OPC or inorganic inverse opal photonic crystal (IOPC) structures have attracted enormous attention in the field of PBG materials and for many other applications. ${ }^{\mathbf{1 3 - 2 0}}$ One of the major issues however is in finding a technique to assemble such opals on a macroscopic length scale.

In general, synthetic opals, which can also be used as templates for the preparation of IOPC structures, typically consist of uniform particles capable of forming periodicities with length scales of 150 to $350 \mathrm{~nm}$. Much effort has been spent in order to improve the macroscopic arrangement of colloidal spheres into an ordered three-dimensional OPC structure. Many techniques exist for this purpose, e.g., based on sedimentation and colloidal crystallization and, in particular, template-directed approaches. ${ }^{21}$ Typically, large-area opals can be prepared by drying ${ }^{22-24}$ or spin coating ${ }^{2526}$ of the particle dispersions. Nevertheless, high quality OPCs with almost perfect three-dimensional particle order remain a significant challenge. A more recent technique for particle ordering focuses on the melt-shear organization of core-shell particles in the absence of any solvent. This route involves the compression of monodisperse hard core-soft shell particles between the plates of a moderately hot press. In recent years, this technique has been further optimized by using a combination of extrusion, rolling and edge-induced rotational shearing steps, providing access to mono-domain bulk-ordered films on the multi-meter 
length scale. ${ }^{27-29}$ Large-area self-supporting OPC films can be obtained..$^{30-34}$ Compared to other well-established methods for the preparation of polymeric opal films, this method is very fast and yields ordered free-standing OPC films in one single step. Until now, this technique was limited to pure organic particle systems featuring a rather low refractive index contrast between the polymer core and the organic matrix material.

Here we report a novel and convenient protocol for the preparation of stimuli-responsive polymer IOPC films on macroscopic length scales. Hybrid core-interlayer-shell (CIS) particles featuring silica cores and surface-anchored responsive polymer shells are prepared. These inorganic/organic particles can be arranged into highly organized hybrid OPC films by using the melt-shear organization technique followed by removing the silica core particles to obtain polymer IOPC structures. This universal methodology for the preparation of IOPC films is exemplarily applied to two different thermoresponsive polymers as matrix-forming materials, poly( $N$-isopropylacrylamide) (PNIPAM) and poly(diethylene glycol methylether methacrylate) (PDEGMEMA). The obtained films are characterized to reveal their regular particle order using atomic force microscopy (AFM) and scanning electron microscopy (SEM) and in terms of their optical response upon mechanical stress, temperature change and solvent response by using UVVis reflection spectroscopy. Expanding this powerful melt-shear organization technique to inorganic particle core materials featuring smart soft-shell polymers is shown to enhance the field of polymer IOPC film preparation in terms of up-scaling strategies and convenient accessibility to novel photonic materials as sensing platforms.

\section{Experimental section}

\subsection{Materials and methods}

Ethyl acrylate (EA), butanediol diacrylate (BDDA) and Irgacure 184 were obtained from BASF SE, methyl methacrylate (MMA) and allyl methacrylate (ALMA) from Evonik Röhm GmbH and Dowfax 2A1 from Dow Chemicals. Diethylene glycol methylether methacrylate (DEGMEMA) and $N$-isopropylacrylamide (NIPAM) were purchased from Sigma Aldrich. All other chemicals were purchased from VWR and Sigma Aldrich and used as received if not otherwise mentioned. Prior to use in emulsion polymerization protocols, the stabilizer hydroquinone monomethylether was removed from EA and MMA monomers. For this purpose, EA and MMA were extracted with $1 \mathrm{M}$ sodium hydroxide solution, washed with water until the solution was neutral followed by drying over sodium sulfate. Silica@PMMA@P(NIPAM-co-MMA) core-interlayer-shell particles were synthesized as described elsewhere. ${ }^{38}$

Transmission electron microscopy (TEM) was performed on a Zeiss EM10 at an operating voltage of $60 \mathrm{kV}$. Scanning electron microscopy (SEM) was performed on a Philips XL30 FEG at an operating voltage of $20 \mathrm{kV}$. Atomic force microscopy (AFM) measurements were performed in the PeakForce tapping mode $^{35}$ using a Dimension Icon atomic force microscope (Bruker AXS, Santa Barbara, CA) and Scanasyst Fluid cantilevers (spring constant $\approx 0.7 \mathrm{~N} \mathrm{~m}^{-1}$ ). The maximum force exerted to the sample was set to $5 \mathrm{nN}$ with an oscillation amplitude of 300 $\mathrm{nm}$ resulting in tip indentation depths into the sample surface of approximately $2 \mathrm{~nm}$ - sufficient to extract the local elasticity of the sample applying the DMT model ${ }^{36}$ to the force-versusseparation data. For dynamic light scattering (DLS) measurements of the particle dispersions a Nanophox photon crosscorrelation spectrometer (Sympatec) was used. The experiments were carried out at an angle of $90^{\circ}$ at $25{ }^{\circ} \mathrm{C}$. Temperaturedependent DLS experiments were carried out with a set-up based on a He-Ne laser $(\lambda=632.8 \mathrm{~nm})$ as the light source. Polarization of the primary beam was defined by a GlanThompson prism. Scattered beam polarization was analysed in vertical-vertical geometry. Scattered intensity was detected with an optical fibre coupled to two avalanche photodiodes that were used in cross-correlation. The intensity autocorrelation functions, calculated with ALV 5000 software for both photodiodes, were measured in steps of $20^{\circ}$ of the scattering angle $\theta$. The angular range was from $50^{\circ}$ to $130^{\circ}$. Measurement time for each angle was $5 \mathrm{~min}$. The measurements were performed in the temperature range from $5{ }^{\circ} \mathrm{C}$ to $20{ }^{\circ} \mathrm{C}$ using cylindrical cuvettes (Hellma) for the samples in a temperature controlled index matching bath. The obtained correlation functions were analysed using the cumulant method. The obtained relaxation times vary with scattering vector $q=4 \pi n / \lambda \sin (\theta / 2)$ as $\tau \approx 1 / q^{2}$. Here $n$ is the refractive index. The correlation time $\tau$ is related to the translational diffusion coefficient $D$ as $D=1 /\left(\tau q^{2}\right)$. The expected linear variation of $1 / \tau$ with $q^{2}$ was well represented by our data. By using the Stokes-Einstein relation, a hydrodynamic diameter $d_{\mathrm{h}}=k_{\mathrm{B}} T / 3 \pi \eta D$ could be calculated, with $k_{\mathrm{B}}, T$, and $\eta$ as the Boltzmann constant, the absolute temperature, and the solvent viscosity, respectively. Thermal properties of the silica@polymer CIS particles were characterized using a differential scanning calorimeter (DSC) (Mettler Toledo DSC-1) in the temperature range from $-50{ }^{\circ} \mathrm{C}$ to $125{ }^{\circ} \mathrm{C}$ for silica@ PEA@P(DEGMEMA-co-EA) CIS particles and from $20{ }^{\circ} \mathrm{C}$ to $180^{\circ} \mathrm{C}$ for silica@PMMA@P(NIPAM-co-MMA) CIS particles with a heating rate of $10 \mathrm{~K} \mathrm{~min}^{-1}$. Reflection spectra were recorded using a Vis-NIR fibre spectrophotometer (USB 2000, Ocean Optics). For the reflection measurements a deuterium/tungsten halogen lamp (DT mini 2, Ocean Optics) was used. Reflection measurements in water and water-diethylene glycol (DEG) mixtures were carried out at normal light incidence $\left(\theta=90^{\circ}\right)$. Solvent- and temperature-induced colour shifts of inverse opal photonic crystal (IOPC) films in water and water-DEG mixtures were tracked after fixing the film with sticky tape on the bottom of a double-wall beaker. Film cooling and heating were carried out using an external cryostat (Thermo Haake). For the temperature-dependent measurements of the swelling process in the temperature range from $15.0{ }^{\circ} \mathrm{C}$ to $0.0{ }^{\circ} \mathrm{C}$, spectra were recorded at intervals of $2.5{ }^{\circ} \mathrm{C}$. In order to ensure a constant temperature of the solvent and the opal film, UV-Vis spectra were recorded after $5 \mathrm{~min}$ of waiting time. Reflection measurements as a function of strain $\varepsilon$ were carried out at normal light incidence $\left(\theta=90^{\circ}\right)$. For this purpose, the straindependent optical properties of a $2 \times 1 \mathrm{~cm}$ strip were measured in situ by homogeneous uniaxial stretching in a water bath by using a custom-made sample holder. 


\subsection{Synthesis and functionalization of silica core particles}

Silica particle dispersions in ethanol with a final silica solid content of $2.5 \mathrm{wt} \%$ were prepared following the procedure described by van Blaaderen et al. ${ }^{37} 1.3 \mathrm{~L}$ of this ethanolic dispersion was mixed with $1.6 \mathrm{~mL}$ of 3-methacryloxypropyltrimethoxysilane (MEMO), and then diluted with $5 \mathrm{~mL}$ ethanol. The mixture was slowly heated to $60{ }^{\circ} \mathrm{C}$ and stirred for $1 \mathrm{~h}$ and ammonia was removed under reduced pressure. Then water and ammonia were removed by azeotropic distillation of the ethanol-water mixture at $60{ }^{\circ} \mathrm{C}$, while the volume was kept constant by continuous addition of ethanol. When the dispersion was free of water and ammonia, the volume was reduced to $350 \mathrm{~mL}$ at $60{ }^{\circ} \mathrm{C}$. In order to transfer the particles into an aqueous dispersion medium, a solution of $50 \mathrm{mg}$ sodium dodecyl sulfate (SDS) in $100 \mathrm{~g}$ of water was added and ethanol was removed by azeotropic distillation, while the volume was kept constant by continuous addition of water. When the dispersion was free of ethanol, the volume was reduced to 130$160 \mathrm{~mL}$. The final MEMO-functionalized silica (silica particle dispersions in water revealed a solid content of 22.1 wt\% (silica particles with an average diameter of $230 \mathrm{~nm}$ ), 20.6 wt\% (silica particles with an average diameter of $199 \mathrm{~nm}$ ) and $17.8 \mathrm{wt} \%$ (silica particles with an average diameter of $172 \mathrm{~nm}$ ), respectively.

\subsection{Synthesis of silica@PEA@P(DEGMEMA-co-EA) CIS particles}

Under argon, a $250 \mathrm{~mL}$ flask equipped with a stirrer and a reflux condenser was filled at $75{ }^{\circ} \mathrm{C}$ with the silica dispersion (110.5 $\mathrm{g}$ of silica particles with an average diameter of $230 \mathrm{~nm}, 118.6 \mathrm{~g}$ of silica particles with an average diameter of $199 \mathrm{~nm}$, or $137.2 \mathrm{~g}$ of silica particles with an average diameter of $172 \mathrm{~nm}$ ), then $68 \mathrm{mg}$ Dowfax 2A1 were added and the particle dispersion was diluted with water to a total mass of $150.0 \mathrm{~g}$. After heating to $75{ }^{\circ} \mathrm{C}$, the polymerization was initiated by adding a solution of $50 \mathrm{mg}$ sodium peroxodisulfate (SPS) in $5 \mathrm{~g}$ water. After $10 \mathrm{~min}$, a monomer emulsion containing $0.2 \mathrm{~g}$ ALMA, $1.02 \mathrm{~g}$ EA, $0.034 \mathrm{~g}$ Dowfax 2A1, $0.04 \mathrm{~g} \mathrm{KOH}$ and $14.65 \mathrm{~g}$ water was added continuously over a period of $1 \mathrm{~h}$ (silica@PEA). After $15 \mathrm{~min}$ of reaction time, a solution of $10 \mathrm{mg}$ SPS in $5 \mathrm{~g}$ water was added. After an additional $10 \mathrm{~min}$, a monomer emulsion of $8.25 \mathrm{~g}$ DEGMEMA, $2.75 \mathrm{~g}$ EA, $0.035 \mathrm{~g}$ SDS and $20.5 \mathrm{~g}$ water was added continuously over a period of $1.8 \mathrm{~h}$ (silica@ PEA@P(DEGMEMA-co-EA)). After an additional $60 \mathrm{~min}$, the product was cooled to room temperature.

\subsection{Preparation of silica@polymer hybrid OPC and polymer IOPC films}

For the preparation of silica@PEA@P(DEGMEMA-co-EA) opal photonic crystal (OPC) films, the latex was dried at $40{ }^{\circ} \mathrm{C}$. The precursor powder was mixed with $0.05 \mathrm{wt} \%$ carbon black (Special Black 4, Degussa), 1 wt\% benzophenone, 1 wt\% Irgacure 184 and $3 \mathrm{wt} \%$ BDDA in a microextruder (micro1, DSM Research) at $80^{\circ} \mathrm{C}$. A $1 \mathrm{~g}$ portion of the mixture was covered with the PET film and heated to $80{ }^{\circ} \mathrm{C}$ between the plates of a laboratory press (300E, Dr Collin). Melt flow was induced by applying a pressure of 150 bar for $3 \mathrm{~min}$, resulting in OPC films of about $10 \mathrm{~cm}$ in diameter and $150 \mu \mathrm{m}$ thickness. For subsequent cross-linking, the films were irradiated with a mercury lamp (UV Cube 2000, Dr Hoenle) with an output power of 1000 $\mathrm{W}$. The PET-covered films were treated at a distance of $10 \mathrm{~cm}$ for $2 \mathrm{~min}$ from both sides. The preparation of silica@PMMA@P(NIPAM-co-MMA) OPCs has been reported elsewhere. ${ }^{38}$

For the preparation of polymer IOPC films, the resulting silica@polymer hybrid OPC films were etched with hydrofluoric acid (HF, $\sim 2 \mathrm{wt} \%$ in water) for 3 days in order to completely remove the silica cores. Afterwards the IOPC films were washed several times and stored in deionized water.

\section{Results and discussion}

In general, soft opal photonic crystal (OPC) films can be obtained from core-interlayer-shell (CIS) particles consisting of a hard core and a surface-anchored soft poly(meth)acrylate shell featuring a low glass transition temperature $\left(T_{\mathrm{g}} \leq 0{ }^{\circ} \mathrm{C}\right)$ by using the powerful melt-shear organization technique. ${ }^{39}$ The resulting opal films are mechanically stable due to the covalent linkage between the cores and the matrix material. But as a second requirement for the melt-shear process, the particle cores must be comparably hard in order to prevent core deformation during melt-shear organization to form highly ordered OPC films. Compared to reports described previously, inhomogeneous opal films were obtained, if poly(meth)acrylates featuring glass transition temperature above room temperature $\left(T_{\mathrm{g}} \geq 20\right.$ $\left.{ }^{\circ} \mathrm{C}\right)$ were used. Here we report that this powerful melt-shear organization technique is not limited to pure organic hard coresoft shell polymer architectures. Basically, the same procedures can be applied on silica@polymer hybrid CIS particles. In order to prepare homogeneous opal films featuring a low or high $T_{\mathrm{g}}$ polymer matrix, silica core particles were used as the starting point and copolymers of poly( $N$-isopropylacrylamide) (PNIPAM) or poly(diethylene glycol methylether methacrylate) (PDEGMEMA) were proven to be a good choice as a shell-forming material for the preparation of hybrid silica@polymer OPC films. In contrast to previously described particle systems, the use of silica as a core material for the synthesis of CIS particles is particularly advantageous: the synthesis of silica particles is well established since 1968 by Stöber, ${ }^{\mathbf{4 0}}$ and silica particles are easy to handle because they do not need the presence of stabilizing agents, e.g., surfactants or surface-anchored polymers to prevent aggregation. Moreover, the rigid silica particles can be functionalized with a high $T_{\mathrm{g}}$ but meltable shell with the possibility for subsequent melt-shear organization in order to form OPC films of silica cores embedded in a hard poly(meth) acrylate matrix. In addition, silica can be easily removed by etching with hydrofluoric acid (HF) after the OPC film formation leading to inverse opal photonic crystal (IOPC) films featuring a well-defined pore structure. The following chapter is divided into different sections introducing the universal methodologies for the generation of polymer IOPC films by using the melt-shear organization technique. First, the general synthesis 
strategy for the formation of uniform hybrid silica@polymer CIS particles is discussed followed by introducing the meltshear organization of the novel particles into hybrid OPC and polymer IOPC films. Beyond these general preparation protocols and film characterization, the stimuli-responsiveness of the newly prepared IOPC films with respect to mechanical stress-, solvent- and temperature-induced structural colour changes is presented.

\subsection{Bottom-up fabrication and characterization of hybrid silica@polymer CIS particles}

For the preparation of the silica@polymer CIS particles described here a combination of the well-established Stöber process and convenient semicontinuous and stepwise emulsion polymerization was used. The silica@polymer CIS particles were synthesized in four main steps as illustrated in Fig. 1: first silica particles were synthesized (Stöber process) followed by a functionalization step. Afterwards a cross-linked interlayer is introduced followed by coating with the meltable outer shell (emulsion polymerization). For the synthesis of hard-soft silica@polymer CIS particles P(DEGMEMA-co-EA) as a soft shell material was used, which was grafted via an ALMA-containing PEA interlayer. To prove the universality of our method described here for soft and hard OPC formation and to compare with the best characterized thermo-responsive polymer in the literature, a copolymer of $N$-isopropylacrylamide and methyl methacrylate (P(NIPAM-co-MMA) as a hard shell polymer featuring an ALMA-containing PMMA interlayer was investigated as well.

The silica cores were prepared from tetraethoxysilane (TEOS) in ethanol as described by van Blaaderen et al. ${ }^{37}$ Corresponding dynamic light scattering (DLS) measurements (Fig. S1 $\dagger$ ) and transmission electron microscopy (TEM) measurements (Fig. S2) are given in ESI $\dagger$. The silica core particles were functionalized with 3-methacryloxypropyltrimethoxysilane (MEMO) to compatibilize the silica surface for the polymer shell growth. Afterwards ethanol was completely substituted by water through distillation without isolating the particles. The resulting silica dispersion in water could be introduced in an emulsion polymerization process to form a cross-linked interlayer by the continuous addition of EA and ALMA for hard-soft CIS particles or MMA and ALMA for hard-hard CIS particles. Finally either EA and DEGMEMA or MMA and NIPAM were added to obtain a film formed of soft or hard shells, respectively. The successful generation of the CIS architecture was followed by DLS measurements (Fig. S3-S5†) evidencing an increase of the particle diameter after each synthesis step. The PDEGMEMA-containing CIS particles were additionally investigated by using TEM measurements (Fig. S6 and S7 $\dagger$ ). Fig. 2 exemplarily shows respective TEM images of silica@ PEA@P(DEGMEMA-co-EA) particles starting from $230 \mathrm{~nm}$ silica cores after key steps of the applied synthetic protocol.
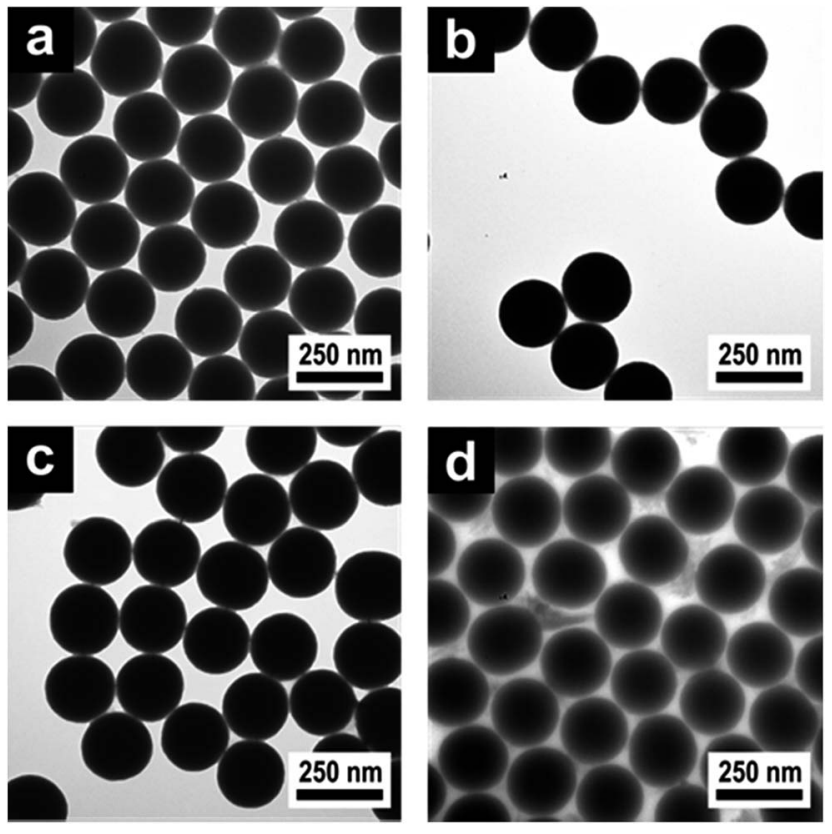

Fig. 2 TEM images of (a) $230 \mathrm{~nm}$ silica core particles, (b) functionalized silica ca@PEA@P(DEGMEMA-co-EA) CIS particles. stöber process

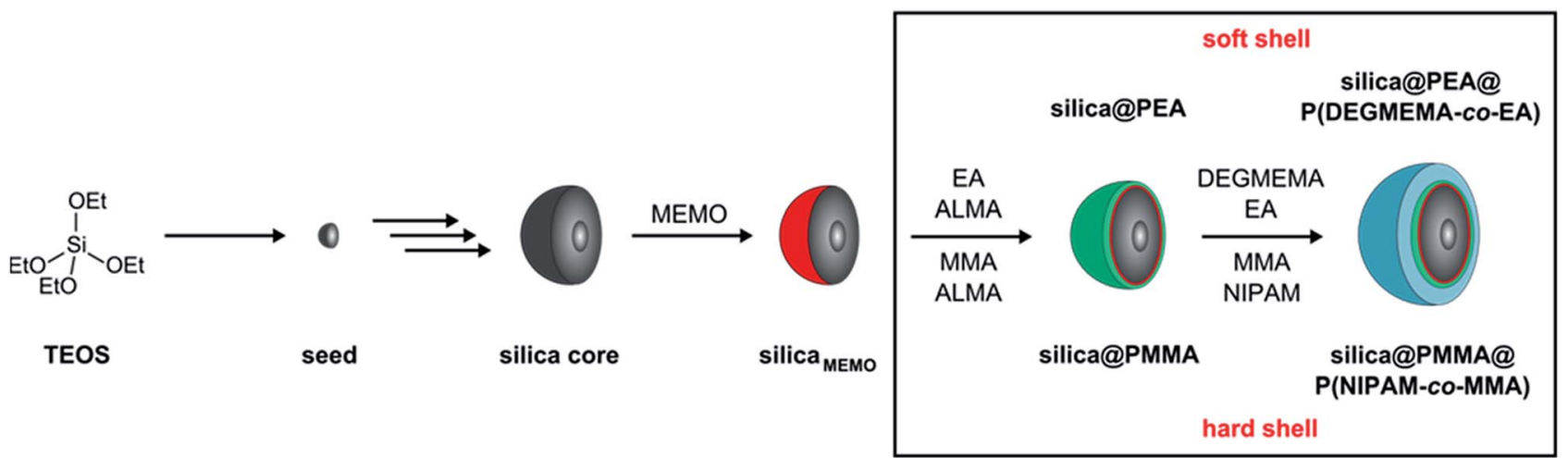

Fig. 1 Stepwise preparation of hard-hard and hard-soft silica@polymer hybrid CIS particles by using the Stöber process and emulsion polymerization protocols. 
From the TEM images it could be confirmed that monodisperse and spherical silica cores (Fig. 2a), separated func-

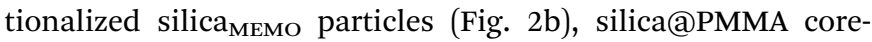
interlayer particles (Fig. 2c) and finally silica@ PEA@P(DEGMEMA-co-EA) CIS particles (Fig. 2d) were formed. As can be concluded from the TEM image of the final silica@ PEA@P(DEGMEMA-co-EA) particles, particle coalescence occurred when the CIS particles were dried on the supporting grid due to the soft shells and the particles tended to form ordered hexagonal arrangements already at room temperature.

Similar products of silica@PEA@P(DEGMEMA-co-EA) particles were synthesized starting from $199 \mathrm{~nm}$ and $172 \mathrm{~nm}$ silica cores, respectively. The average diameters obtained by TEM $\left(d_{\text {TEM }}\right)$ and hydrodynamic diameters $\left(d_{\text {DLS }}\right)$ obtained by DLS measurements of all particle batches prepared are summarized in Table $\mathrm{S} 8 \dagger$ and were in excellent agreement with expectations. The successful synthesis of silica@PMMA@P(NIPAM-co-MMA) has been recently reported elsewhere ${ }^{38}$ but is also mentioned here for the sake of completeness and for proving the generality of the applied synthetic protocol. The average diameters $d_{\text {TEM }}$ and $d_{\text {DLS }}$ of silica@PMMA@P(NIPAM-co-MMA) that were prepared from $160 \mathrm{~nm}$ silica cores are also given in Table S8. $\dagger$

In order to investigate the thermo-responsiveness of these hybrid CIS particles, the reversible swelling and shrinking behaviour of the shell polymer in water was studied. The thermosensitivity of PNIPAM-containing hybrid core-shell particles has already been reported in several publications and was found to be repeatedly addressable. ${ }^{\mathbf{4 1 - 4 3}}$ To demonstrate the temperature-induced volume phase transition of surface-anchored shell polymers of the novel silica@PEA@P(DEGMEMA-co-EA) particles, the particle dispersion was repeatedly brought above or below the LCST of P(DEGMEMA-co-EA) and changes of the hydrodynamic particle diameter $\left(d_{\mathrm{h}}\right)$ were followed by using temperature-dependent DLS measurements. In detail, the particles were first cooled from $20.0{ }^{\circ} \mathrm{C}$ to $5.0{ }^{\circ} \mathrm{C}$ and again heated up to $20.0{ }^{\circ} \mathrm{C}$. Fig. 3 shows $d_{\mathrm{h}}$ of the particles as a function of temperature for the cooling and heating run.

As expected, there is a strong change in size induced by the change in temperature. The PDEGMEMA-containing hybrid particles revealed a large increase of $d_{\mathrm{h}}$ from initially $325 \mathrm{~nm}$ at $20{ }^{\circ} \mathrm{C}$ to $408 \mathrm{~nm}$ after cooling to $5{ }^{\circ} \mathrm{C}$. Similar to PS@PEA@P(DEGMEMA-co-EA) CIS particles, ${ }^{39}$ the transition is also fully reversible for these new hybrid materials. From Fig. 3 it can be concluded that the swelling of the P(DEGMEMA-co-EA) shell can clearly be influenced by temperature and the silica cores do not significantly alter the swelling behaviour of the polymer shell.

The silica@PEA@P(DEGMEMA-co-EA) as well as silica@PMMA@P(NIPAM-co-MMA) have been further characterised by differential scanning calorimetry (DSC) to estimate the glass transition of the final shell polymers (Fig. S9 and 10 ${ }^{\dagger}$ ). As expected, the measured $T_{\mathrm{g}}$ value of P(NIPAM-co-MMA) $\left(T_{\mathrm{g}}=\right.$ $+112{ }^{\circ} \mathrm{C}$ ) is significantly higher than the $T_{\mathrm{g}}$ value found for $\mathrm{P}$ (DEGMEMA-co-EA) $\left(T_{\mathrm{g}}=-20^{\circ} \mathrm{C}\right)$. As the processing of the hard-soft CIS particles usually takes place at temperatures of about $20-140{ }^{\circ} \mathrm{C}, \mathrm{P}$ (NIPAM-co-MMA) seemed to be a good choice to form hard OPC films. However, at the higher processing temperatures changes of the mechanical and rheological

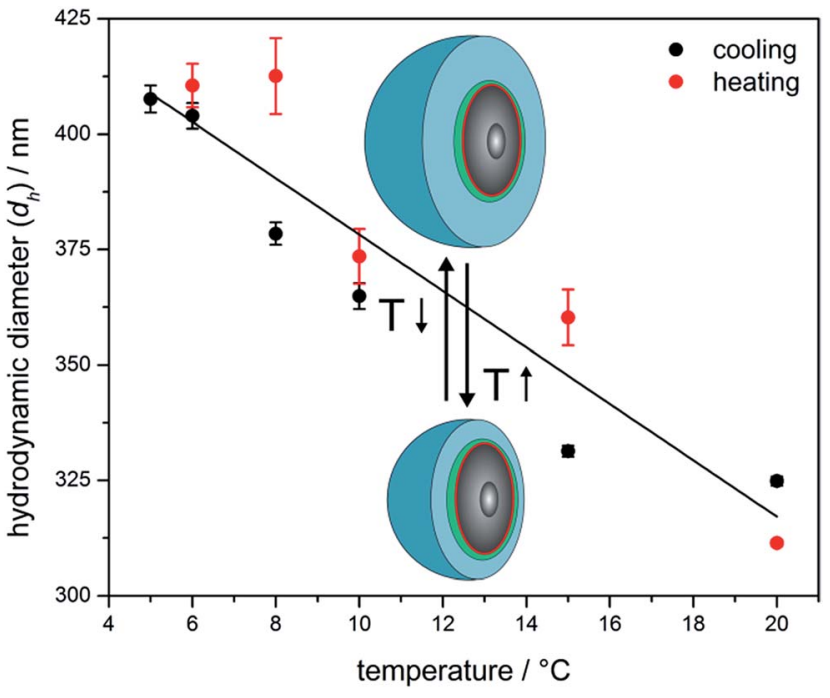

Fig. 3 Temperature-dependent DLS investigations of silica@PEA@P(DEGMEMA-CO-EA) particles with $230 \mathrm{~nm}$ silica cores (the solid line is a guide for the eye).

properties of the shell polymer and the viscoelastic melt have to be critically considered. The melt-shear organization as well as different cross-linking approaches of these hybrid silica@polymer CIS particles are discussed in the ensuing section.

\subsection{Preparation and characterization of hybrid OPC and IOPC films}

Typically, the fabrication of polymer IOPC films requires a multi-step templating strategy, which comprises the (self) assembly of colloidal silica spheres into colloidal crystals, the infiltration of the structure with a solution of radical initiator, monomer and cross-linker, followed by thermo- or photo-polymerization and removal of the colloidal crystal template by etching with HF. ${ }^{4-48}$ However, problems have previously occurred by this method since the infiltration and polymerization stage of the process lead to cracking of the structures and the prepared IOPCs are neither compact nor monocrystalline.

In our approach, compact, stable and monocrystalline colloidal crystals that are embedded in a polymer matrix are obtained by the method of melt-shear..$^{3,34,49-52}$ Inspired by this work, we now conceived the technique for the novel thermoresponsive silica@PEA@P(DEGMEMA-co-EA) and silica@PMMA@P(NIPAM-co-MMA) hybrid CIS particles to prepare hybrid OPC and polymer IOPC films by applying melt-shear organization, cross-linking (xlink) and subsequent etching of the silica cores, thus avoiding the infiltration stage and the cracking of the films. After etching the silica cores, the resulting IOPC films consist only of crystalline arranged pores embedded in a polymer matrix. A scheme of the preparation process is depicted in Fig. 4.

Prior to melt-shear organization, the silica@polymer CIS particles were separated from their dispersion by drying at $40{ }^{\circ} \mathrm{C}$. After complete evaporation of water, the silica@ PEA@P(DEGMEMA-co-EA) particles had the consistency of a soft and sticky solid, while the silica@PMMA@P(NIPAM-co- 
MMA) particles featured the consistency of a solid powder at room temperature. Afterwards, both products could be mixed with different cross-linking agents by using extrusion at elevated temperatures, whereby the shells of the CIS particles merged into viscous liquids forming a continuous molten matrix. In order to enhance the reflection colours of corresponding OPCs and IOPCs, 0.05 wt $\%$ of carbon black powder were added during extrusion, which has been found to dramatically enhance the perceived reflection colour due to spectrally resonant scattering inside the OPC structure without affecting the lattice quality. ${ }^{53}$

The soft silica@PEA@P(DEGMEMA-co-EA) precursor could be processed at a relatively low temperature of $80^{\circ} \mathrm{C}$, which made it possible to use the temperature-sensitive bifunctional monomer butanediol diacrylate (BDDA) as a cross-linker and commercially available photoinitiators benzophenone and Irgacure 184 (weight ratio: $95: 3: 1: 1$ ), which had already been shown to be very effective for subsequent photocross-linking of polymer OPCs. ${ }^{39,49,50}$ In the case of silica@PMMA@P(NIPAM-coMMA) particles even higher processing temperatures were necessary due to the higher $T_{\mathrm{g}}$ value of the shell polymer $\left(T_{\mathrm{g}}=\right.$ $+112{ }^{\circ} \mathrm{C}$ ). For that reason the precursor material had to be processed in the extruder at $180{ }^{\circ} \mathrm{C}$. Since BDDA was not suitable for this processing temperature, the bifunctional monomer $N, N^{\prime}$-(1,2-dihydroxy-ethylene)bisacrylamide (DHEBA) in combination with benzophenone and Irgacure 184 (weight ratio: $88: 10: 1: 1)$ were chosen as cross-linking agents to prevent premature thermal cross-linking that would hinder the meltshear organization. DHEBA monomer starts to melt at $156{ }^{\circ} \mathrm{C}$, so all components formed homogeneous mixtures at the processing temperature of $180{ }^{\circ} \mathrm{C}$. The extruded strands were then heated to the respective processing temperature between the plates of a laboratory press, in which the meltable shells merge into a continuous molten matrix. Then melt-shear was induced by applying pressure perpendicular to the plates and the silica cores arranged themselves in the parabolic flow field into stacked hexagonal layers resulting in a precise close-packed arrangement of the silica cores embedded in the polymer matrix. Prior to the formation of IOPCs by etching with HF, the formation of a mechanically stable hybrid OPC film was achieved by subsequent cross-linking reactions of the matrix polymer. During UV irradiation the free polymer chains in the matrix material were linked with embedded BDDA or DHEBA monomer, respectively, generating a dense network. Afterwards, the silica cores were removed with $\mathrm{HF}$ aqueous solution ( $\sim 2 \mathrm{wt} \%$ ), and the corresponding IOPC hydrogel was thoroughly washed and stored in deionized water. To ensure that silica cores were completely removed after etching and polymer IOPCs were on hand, cross-sections of the IOPCs were prepared for SEM investigations. Fig. 5 shows respective AFM and SEM images of corresponding OPC and IOPC films.

From the AFM PeakForce tapping image showing a map of the local elasticity of the surface of the soft OPC film (Fig. 5a) it can be confirmed that the film contains closely packed hexagonally arranged layers of silica spheres that are aligned parallel to the sample surface. The top layer of the hard silica cores is exposed in the AFM image, because the spheres are stiffer than the soft surrounding polymer matrix. The elastic modulus of the silica spheres is approximately $300 \mathrm{MPa}$, i.e. softer than expected due to a thin polymer film covering the spheres, thus reducing the effective modulus. The spherical silica cores embedded in the polymer matrix can also be seen in the SEM image of a cross-section of the film (Fig. 5b). After etching with HF the porous character of the soft IOPC films could be proven by SEM investigations of film cross-sections, which show that the matrix material is completely pervaded with pores. A comparison of Fig. $5 \mathrm{~b}$ and c confirms that the initial structure is replicated and a microscopic pore structure appeared after inversion. It should be noted that after etching the silica and subsequent drying for SEM imaging, the surface and the majority of the pores were closed because of the collapse of the soft matrix during drying. Therefore, the resulting IOPC film was directly used for SEM imaging, and no more extra treatments were performed for SEM sample preparation.

For the hard OPCs the precise arrangement of the surface parallel (111) planes could be seen in SEM investigations of the corresponding film surface after etching the matrix material with oxygen plasma (Fig. 5d). Silica cores arranged in hexagonal layers of the (111) planes are evidenced and it is clear that the OPC film has a close-packed structure with long-range regularity. In the SEM image of the IOPC film surface after etching with HF (Fig. 5e), an almost perfectly ordered hexagonal arrangement of air pores embedded in the polymer matrix is visible and the inverted IOPC structure reproduces the lattice of

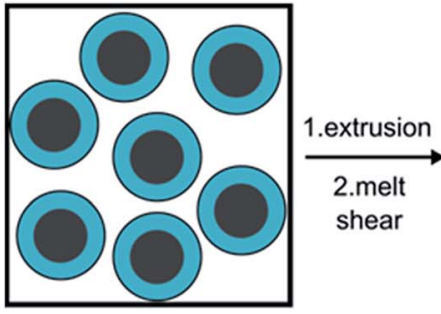

silica@polymer particle dispersion

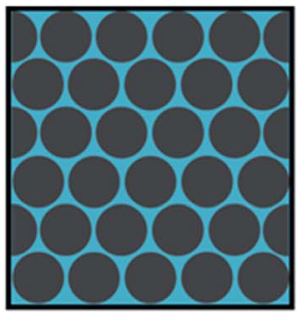

silica@polymer hybrid OPC

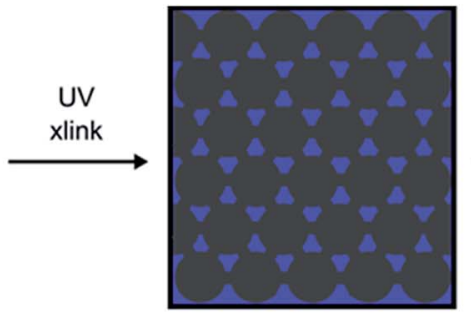

xlinked hybrid OPC

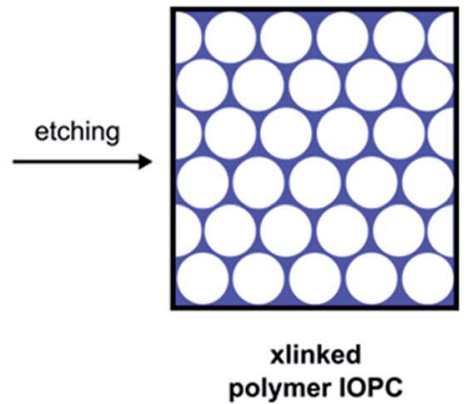

Fig. 4 Processing of the CIS particles into close-packed, cross-linked (xlinked) silica@polymer hybrid opal photonic crystal (OPC) and polymer inverse opal photonic crystal (IOPC) films. 

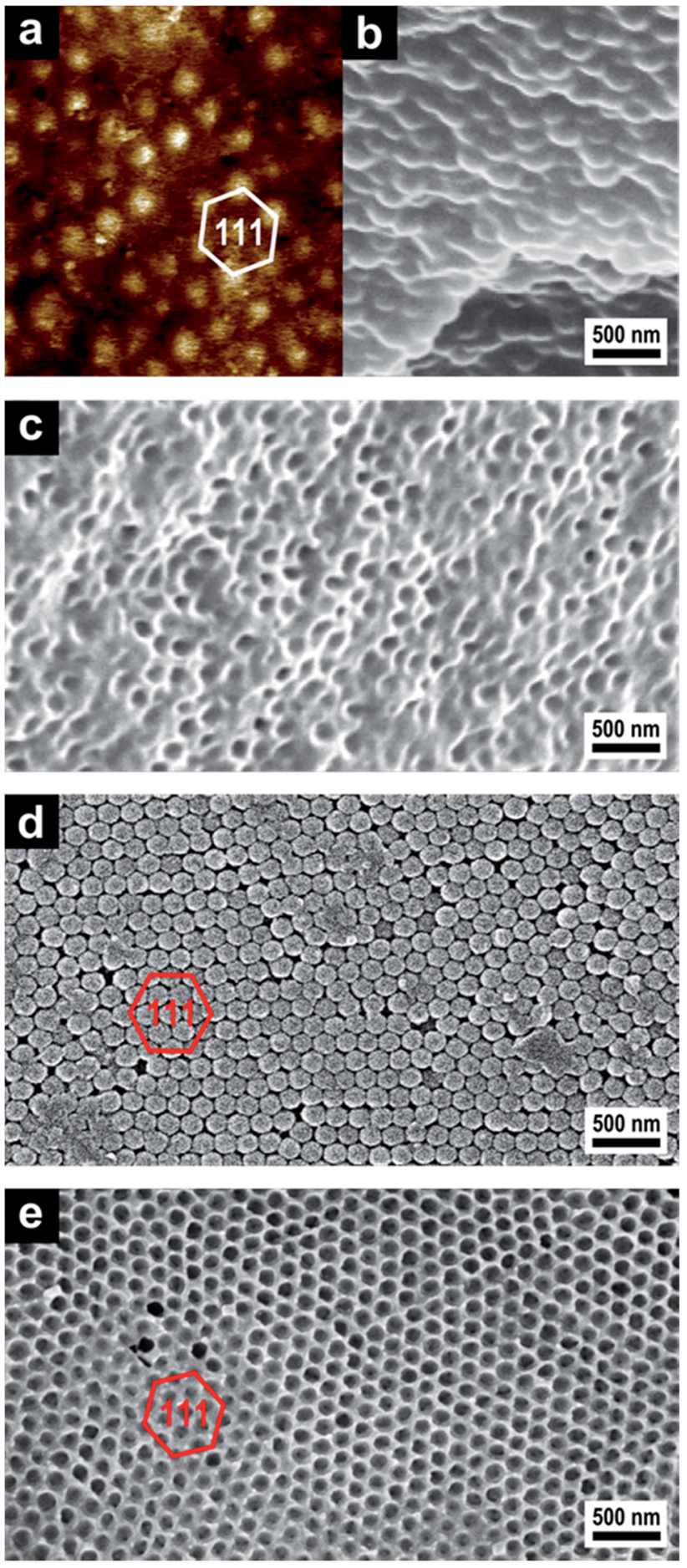

Fig. 5 (a) PeakForce tapping AFM image revealing the local elasticity of soft silica@PEA@P(DEGMEMA-CO-EA) OPC. A hexagonally arranged (111) plane of silica particles (light) embedded in the polymer matrix (dark) is clearly visible (color scale: 145-307 MPa). (b) SEM image of a cross-section of silica@PEA@P(DEGMEMA-co-EA) OPC showing silica particles embedded in the polymer matrix. (c) SEM image of a crosssection of P(DEGMEMA-CO-EA) IOPC after etching with HF showing air pores (dark) embedded in the polymer matrix (light). (d) SEM image of the oxygen plasma-etched surface of silica@PMMA@P(NIPAM-coMMA) OPC showing the hexagonally arranged (111) plane of silica spheres. (e) SEM image of the surface of P(NIPAM-cO-MMA) IOPC after etching with HF showing the hexagonally arranged (111) plane of air pores (dark) embedded in the polymer matrix (light). the initial OPC almost completely. It should be noted that the surface of the inverse opal film is open, as shown by the SEM image (Fig. 5e). Additionally, the pores are not connected, but rather are isolated from each other by the matrix material. This is the reason why the next layer cannot be observed under the first layer in the SEM image of the IOPC structure (Fig. 5e).

As can be concluded from Fig. 5, the melt-shear organization of silica@polymer hybrid CIS particles led to regularly ordered OPC films, while the subsequent cross-linking reaction provided inherently stable films which could therefore be completely converted into IOPC structures. Moreover, all of these films featured Bragg reflection colours evidencing the homogeneous domain size.

For that reason, the success of HF etching could also be proven by examining the changes of the Bragg-peak position and peak intensity in UV-Vis reflection measurements. The perceived Bragg peak wavelength $\lambda_{111}$ of the surface parallel (111) plane (stop band) of the films depends on several factors, including the periodicity $a_{111}$ and the average refractive index $n_{\text {eff }}$ of the OPCs and IOPCs and can be well described by combining Bragg's law with Snell's law (eqn (1)), where $\theta$ denotes the angle of incident light:

$$
\lambda_{111}=2 a_{111}\left(n_{\mathrm{eff}}^{2}-\sin ^{2} \theta\right)^{1 / 2}
$$

The average refractive index $n_{\text {eff }}$ can be calculated by considering the refractive indices $\left(n_{\mathrm{i}}\right)$ and the volume fractions $\left(\phi_{\mathrm{i}}\right)$ for both the silica cores respectively the pores and the polymer shells as well as the water content of the IOPC hydrogel under certain conditions according to eqn (2):

$$
n_{\text {eff }}=\sum n_{\mathrm{i}} \phi_{\mathrm{i}}
$$

In addition, it is well known that the intensity of the reflection peaks is proportional to both the magnitude of the refractive index contrast $\Delta n$ and the number of ordered lattice planes that result from the periodic close-packed arrangement. According to eqn (1) the Bragg peak corresponds to the light diffraction from the surface parallel (111) plane, which can be measured perpendicular $\left(\theta=90^{\circ}\right)$ to the sample surface. Because $a_{111}$ is directly proportional to the respective particle core size, the colour of the OPCs and corresponding IOPCs can easily be shifted to lower wavelength colours simply by decreasing the size of the silica cores used for the preparation of CIS particles. To demonstrate effects of the core size and silica etching on the colour and reflection intensity of the films, we prepared OPC and IOPC samples from CIS particles, which differed in the size of the silica cores. Fig. 6 shows reflection spectra as well as photographs of OPCs and IOPCs prepared from CIS particles with $230 \mathrm{~nm}, 199 \mathrm{~nm}, 172 \mathrm{~nm}$, and $160 \mathrm{~nm}$ silica cores before and after etching with HF.

Surprisingly, despite the low refractive index contrast between the silica cores $(n \sim 1.45)$ and the polymer shell $(n \sim$ 1.47), the resultant hybrid OPC samples revealed distinct iridescent colours. Obviously, the films feature well-ordered layers, which was also proven in the UV-Vis reflection spectra in Fig. 6. The hard OPC film showed similar strong reflection 
colours and, as evidenced by the reflection spectra, was as crystalline as the soft OPC films. The colours of the films were furthermore improved after subsequent etching of the silica cores, resulting in brilliant IOPC films that appear green $\left(\lambda_{111}=\right.$ $554 \mathrm{~nm})$, turquoise $\left(\lambda_{111}=495 \mathrm{~nm}\right)$, blue $\left(\lambda_{111}=442 \mathrm{~nm}\right)$ and purple $\left(\lambda_{111}=427 \mathrm{~nm}\right)$, respectively. Two general spectral characteristics have to be noted as the OPC films are inverted: compared to the initial OPC films, the IOPCs reflect very strongly and the intensity of the Bragg peaks increases greatly after inversion (up to threefold), but nevertheless the Bragg peak position changes only slightly. Both effects can be explained as follows: the intensity of the peak increased due to an increase in $\Delta n$, since the silica cores $(n \sim 1.45)$ inside the OPCs are replaced by water featuring a much lower refractive

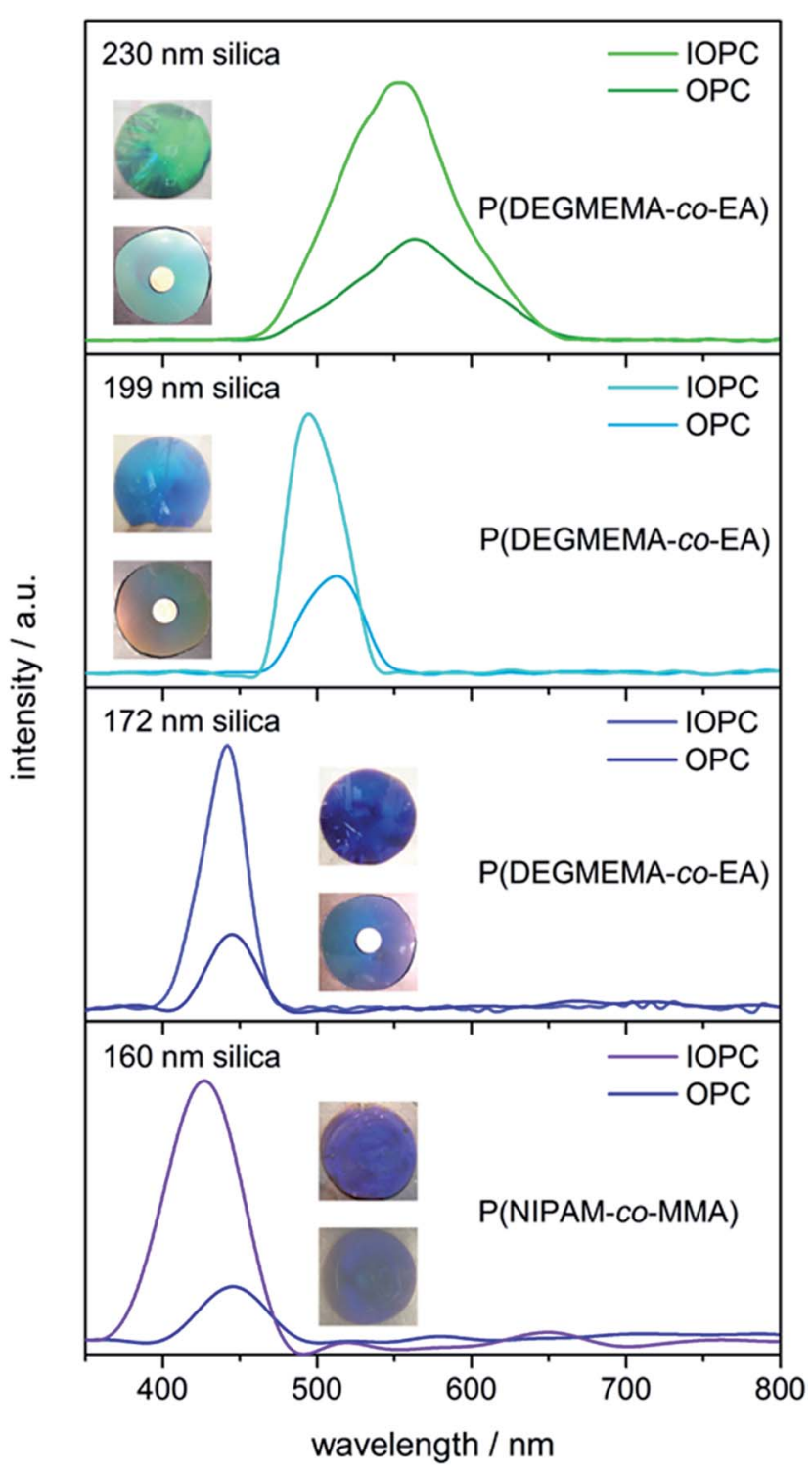

Fig. 6 UV-Vis reflection spectra and photographs of OPC films before and IOPC films after etching with HF, prepared from silica apolymer CIS particles with $230 \mathrm{~nm}, 199 \mathrm{~nm}, 172 \mathrm{~nm}$ and $160 \mathrm{~nm}$ silica cores (from top to bottom), respectively. The upper photographs correspond to the swollen IOPCs, while the other photographs correspond to the bulk OPC films. index $(n \sim 1.33)$. At first glance, one may wonder about the fact that $\lambda_{111}$ shifts only slightly to lower wavelength after inversion, although $n_{\text {eff }}$ of the compact OPC and the porous IOPC films was quite different. This phenomenon is based on the compensation of two different effects. According to eqn (1), $\lambda_{111}$ depends on $n_{\text {eff }}$, which is lower in the IOPCs, but it also depends on $a_{111}$, which is slightly higher in the IOPC films, because they are capable of slight swelling in water already at room temperature forming IOPC hydrogel films.

It can be concluded that well-ordered soft or hard OPC films were accessible from silica@polymer CIS particles by applying the melt-shear organization technique. These films could be completely converted into IOPC hydrogels after etching the silica cores. After inversion, the colour brilliance of the films is strongly enhanced, however, the position of the Bragg peaks changes only slightly due to the swelling of the polymer matrix in water.

\subsection{Mechano-, thermo- and solvatochromic response of inverse opal photonic crystal films}

The Bragg peak positions of the stop bands of IOPCs can be varied in a predictable manner by altering certain variables in eqn (1). Since the P(DEGMEMA-co-EA) IOPCs feature a highly porous structure that is embedded in an elastic and thermoresponsive hydrogel matrix, $a_{111}, n_{\text {eff }}$ and $\Delta n$ can be influenced by applying various stimuli, such as mechanical deformation, IOPC hydrogel infiltration, swelling and deswelling or thermally induced polymer volume phase transitions. Consequently, $\lambda_{111}$ can effectively be modulated. Therefore the optical response of the prepared P(DEGMEMA-co-EA) IOPC film was investigated with regard to its mechano-, solvato- and thermochromic response and its usability for optical sensing applications. The possible stimuli-induced structural changes of the IOPC film are illustrated in Fig. 7.

As depicted in Fig. 7, strain-induced structural colour tuning of the IOPCs mainly depends on the lattice deformation, changing both the symmetry and $a_{111}$ of the lattice. It is worth noting that mechanical stretching of the IOPC embedded in the elastomeric matrix induces an anisotropic change of the lattice constant, which results in a reduced symmetry of the crystal lattice. Since P(DEGMEMA-co-EA) reveals a temperature-sensitive behaviour that undergoes a reversible volume phase transition between a hydrated state and a dehydrated state around its LCST ( $c f$. DLS measurements in Fig. 2), the polymer swells or deswells in water and expands or contracts, so that $a_{111}$ in the IOPC can be reversibly modulated by changing the temperature. Typically, IOPC structures also show tunable diffraction when

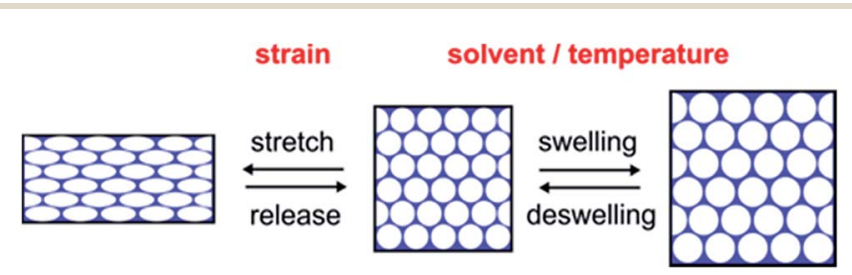

Fig. 7 Reversible strain-, solvent- and temperature-induced structural changes of the polymer IOPC structure. 
they are subjected to solvent infiltration, swelling or deswelling, with the diffraction wavelength dependent on the degree of swelling of the hydrogel and degree of pore infiltration. Generally, the swelling or infiltration with solvents is accompanied by the change of $n_{\text {eff }}$ as well as $\Delta n$ between the filled pores and the matrix material. So if the IOPC structure is repeatedly immersed into a solvent, whose refractive index closely matches that of the matrix material $(\Delta n \sim 0)$, the diffraction intensity can be switched on and off reversibly. To demonstrate this interesting behaviour experimentally, we chose diethylene glycol (DEG, $n \sim 1.45$ ) as the solvent to have almost equal refractive indices.

For the determination of the stimuli-induced structural colour shifts we used the green P(DEGMEMA-co-EA) IOPC film, which was swollen in water, corresponding to a photonic hydrogel film. Since elasticity is given due to matrix crosslinking, the mechanochromic behaviour of the entire film was still available in the swollen state. For the determination of stress-, solvent- and temperature-induced colour shifts, the IOPC film was either stretched up to strains of $\varepsilon=20 \%$ or cooled down from $T=15.0{ }^{\circ} \mathrm{C}$ to $0.0{ }^{\circ} \mathrm{C}$ or the film was immersed in mixed solutions of water and DEG with a decreased water-DEG ratio. The corresponding UV-Vis reflection measurements are shown in Fig. 8.

As strain was increased, a blue-shift of the (111) plane stop band was clearly visible in reflection spectra, while the maximum in the reflection intensity decreased (Fig. 8, left). The blue-shift of $\lambda_{111}$ under increasing strain is based on decreasing $a_{111}(c f$. eqn (1)). Strains of approximately $20 \%$ shifted the stop band by about $45 \mathrm{~nm}$; i.e. the reflected colour changes from its initial green $\left(\lambda_{111}=554 \mathrm{~nm}\right)$ to turquoise $\left(\lambda_{111}=509 \mathrm{~nm}\right)$. We note that the decrease in reflection intensity in Fig. 8 (left) during stretching is due to a loss of crystalline order from the lattice deformation. But when the film was allowed to relax to its original state, the IOPC structure reassembled giving identical position and intensity of the (111) plane stop band and the green film colour was fully recovered (Fig. S11†).
In addition, the temperature-dependent UV-Vis reflection spectra shown in Fig. 8 (right) evidence a temperature-induced swelling accompanied by a stop band shift of slightly more than $15 \mathrm{~nm}$, i.e. a change from green $\left(\lambda_{111}=554 \mathrm{~nm}\right)$ to yellow $\left(\lambda_{111}=\right.$ $572 \mathrm{~nm}$ ) of the Bragg peak under normal light incidence after cooling to $0.0{ }^{\circ} \mathrm{C}$. An accompanying decrease in reflection intensity and reduced visual colour brilliance are seen with the red-shift of $\lambda_{111}$ (Fig. 8, right). Both effects can be explained as follows: at low temperatures, a significant swelling of the IOPC matrix with water takes place while reaching the LCST of $\mathrm{P}$ (DEGMEMA-co-EA). Hence, the volume fraction of water featuring a comparably low refractive index was increased in the matrix material. This led to a lower $\Delta n$ between the water filled pores and the hydrogel matrix causing a decrease in the reflection intensity. Moreover, the temperature-induced swelling caused a volume expansion of the macroscopic film resulting in the increase of $a_{111}$ of pores embedded in the crosslinked matrix and therefore a red-shift of $\lambda_{111}(c f$. eqn (1)). It is important to note that due to the presence of a significant amount of different cross-linking additives, the LCST of the entire IOPC film is expected to further decrease compared to the purified single CIS particle dispersion ( $c f$. DLS measurements Fig. 3). After subsequent heating to $15{ }^{\circ} \mathrm{C}$, the IOPC hydrogel revealed inverted colour shifts and the original IOPC structure was totally restored (Fig. $\mathrm{S} 12 \dagger$ ).

In the next step, the optical response of the IOPC film was investigated for its solvatochromic behaviour and to gain insight into wettability and infiltration properties. It is known that $\mathrm{P}$ (DEGMEMA-co-EA) is hydrophilic and tends to absorb water already at room temperature. But this effect is restrained by adding DEG in water (Fig. 8, middle). When the IOPC film was immersed into mixtures of water-DEG with a decreased water-DEG ratio, changes of the structural colour within a few seconds could be observed and $\lambda_{111}$ was blue-shifted from its original green $\left(\lambda_{111}=554 \mathrm{~nm}\right)$ to turquoise $\left(\lambda_{111}=503 \mathrm{~nm}\right)$ until the water content reached $70 \%$ (Fig. 8, middle). Simultaneously, the peak intensity decreased sharply and, at even higher DEG contents, the reflection peak almost disappeared. The blue-shift
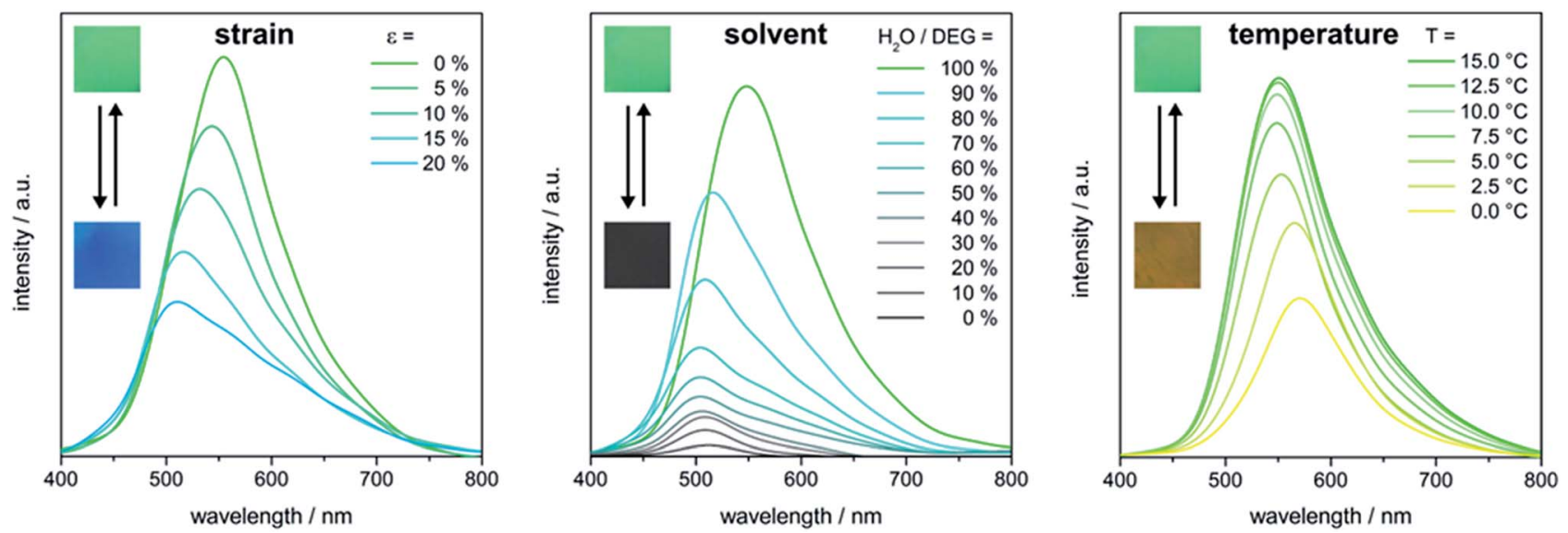

Fig. 8 UV-Vis reflection spectra of the IOPC hydrogel film prepared from silica@PEA@P(DEGMEMA-co-EA) CIS particles with $230 \mathrm{~nm}$ silica cores showing stress- (left), solvent- (middle) and temperature- (right) induced colour shifts (insets: photographs of the IOPC hydrogel film before and after stress-, solvent- and temperature-induced colour tuning). 
of $\lambda_{111}$ can be attributed to the decrease in $a_{111}$ of the IOPC structure due to the collapse of the hydrogel with increasing DEG content. The isotropic change in the volume of the hydrogel caused continuous shifting of $\lambda_{111}$, indicating that the porous IOPC gel maintained its nanostructure. Furthermore, the refractive index of the IOPC matrix changed only slightly when the IOPC is soaked in different water-DEG solutions, however, the infiltration of the pores with different solutions caused a tremendous change in refractive index between 1.33 (pure water) and 1.45 (pure DEG) inside the pores. After complete collapse of the hydrogel network the infiltration of solutions with higher DEG content into the IOPC structure, with the average refractive index rising, led to complete suppression of the reflection peak on the time scale of seconds, providing further evidence for the very fast and efficient wetting and infiltration of the IOPC structure. Since the refractive index of pure DEG closely matched that of the P(DEGMEMA-co-EA) matrix $(\Delta n \sim 0)$, the reflection colour completely disappeared and the film appeared grey (due to incorporated carbon black powder) and transparent in pure DEG. This distinct change in colour demonstrates that the response of the IOPC film to water-DEG is perceptible with the naked eye. After subsequent immersion into pure water, the IOPC hydrogel revealed inverted colour shifts and the original IOPC structure with identical position and intensity of the (111) plane stop band and the green film colour was totally restored within at least one minute (Fig. S13†). To summarize, the intense reflectivity of the IOPC film can be switched easily between "on" and "off" states by alternating immersion into pure DEG and pure water. The easily scalable preparation and the excellent stability of the polymer IOPCs together with the capability of relatively fast on-off switching of the diffraction make these films excellent candidates for security materials and optical sensing applications.

\section{Conclusions}

In conclusion, this work demonstrates a novel feasible approach for using the melt-shear organization technique in order to prepare large-area inorganic/organic opal photonic crystal (OPC) and polymer inverse opal photonic crystal (IOPC) films. For applying this convenient technique, surface immobilization of meltable (stimuli-responsive) polymers at silica core particles was necessary and possible by combining the Stöber reaction and seeded emulsion polymerization protocols. By using these techniques, monodisperse and adjustable particles with average diameters in the range of half the wavelength of visible light were obtained. Poly $(\mathrm{N}$-isopropylacrylamide) (PNIPMA) and poly(diethylene glycol methylether methacrylate) (PDEGEMA) were used as meltable and thermo-responsive shell materials for the core-shell-particles providing access to hybrid OPC films with $10 \mathrm{~cm}$ in diameter with an excellent particle ordering after melt-shear organization. The high order of the hard/soft particles was investigated and evidenced by using atomic force microscopy, SEM and UV-Vis measurements. Thermo-responsiveness of the pristine particles prior to opal film preparation was evidenced by using temperature-dependent dynamic light scattering measurements of the particle dispersion in water. A reversible increase and decrease of about $80 \mathrm{~nm}$ of the average particle diameter were observed in water upon changing the temperature from $20{ }^{\circ} \mathrm{C}$ to $5{ }^{\circ} \mathrm{C}$. This remarkable thermo-responsive behaviour was also observed for the stimuli-responsive polymer IOPC films after etching the silica core particles. Moreover, the IOPC films revealed excellent stretch-tunable properties and solvent response accompanied by a photonic band gap switching. This developed melt-shear ordering technique for inorganic/organic core-shell particle systems for the preparation of hybrid opal and inverse opal polymer films is expected to be a universal platform applicable to a broad range of different materials. Thus such stimuli-responsive photonic band gap materials are promising candidates in the field of optical sensing devices and actuation systems.

\section{Acknowledgements}

The authors want to thank the Landesoffensive zur Entwicklung Wissenschaftlich-ökonomischer Exzellenz (LOEWE Soft Control), UK EPSRC EP/L027151/1, and ERC LINASS 320503 for ongoing financial support of this work. We thank Christina Lederle (Department of Physics, TU Darmstadt) for performing temperature-dependent DLS measurements and Prof. Bernd Stühn for helpful discussion. The authors gratefully acknowledge technical support of Stefano Nieddu for constructing the reflection measurement setup.

\section{Notes and references}

1 Y. Xia, B. Gates, Y. Yin and Y. Lu, Adv. Mater., 2000, 12, 693713.

2 A. P. Hynninen, J. H. Thijssen, E. C. Vermolen, M. Dijkstra and A. van Blaaderen, Nat. Mater., 2007, 6, 202-205.

3 L. Gonzalez-Urbina, K. Baert, B. Kolaric, J. Perez-Moreno and K. Clays, Chem. Rev., 2012, 112, 2268-2285.

4 J. F. Galisteo-Lopez, M. Ibisate, R. Sapienza, L. S. FroufePerez, A. Blanco and C. Lopez, Adv. Mater., 2011, 23, 30-69.

5 C. Fenzl, T. Hirsch and O. S. Wolfbeis, Angew. Chem., Int. Ed., 2014, 53, 3318-3335.

6 J. H. Lee, C. Y. Koh, J. P. Singer, S. J. Jeon, M. Maldovan, O. Stein and E. L. Thomas, Adv. Mater., 2014, 26, 532-569.

7 A. Stein, B. E. Wilson and S. G. Rudisill, Chem. Soc. Rev., 2013, 42, 2763-2803.

8 J. Ge and Y. Yin, Angew. Chem., Int. Ed. Engl., 2011, 50, 14921522.

9 Y. Takeoka, J. Mater. Chem. C, 2013, 1, 6059-6074.

10 C. I. Aguirre, E. Reguera and A. Stein, Adv. Funct. Mater., 2010, 20, 2565-2578.

11 J. M. Weissman, H. B. Sunkara, A. S. Tse and S. A. Asher, Science, 1996, 274, 959960.

12 J. H. Lee, C. Y. Koh, J. P. Singer, S. J. Jeon, M. Maldovan, O. Stein and E. L. Thomas, Adv. Mater., 2014, 26, 532-569.

13 K. R. Phillips, N. Vogel, Y. Hu, M. Kolle, C. C. Perry and J. Aizenberg, Chem. Mater., 2014, 1622-1628. 
14 K. Rhee do, B. Jung, Y. H. Kim, S. J. Yeo, S. J. Choi, A. Rauf, S. Han, G. R. Yi, D. Lee and P. J. Yoo, ACS Appl. Mater. Interfaces, 2014, 6, 9950-9954.

15 J. Zhao, Y. Liu and Y. Yu, J. Mater. Chem. C, 2014, 2, 1026210267.

16 J. Kim, S. A. Bencherif, W. A. Li and D. J. Mooney, Macromol. Rapid Commun., 2014, 35, 1578-1586.

17 J. Cui, W. Zhu, N. Gao, J. Li, H. Yang, Y. Jiang, P. Seidel, B. J. Ravoo and G. Li, Angew. Chem. Int. Ed., 2014, 53, 3844-3848.

18 M. Retsch and U. Jonas, Adv. Funct. Mater., 2013, 23, 53815389.

19 J. Zhou, H. Li, L. Ye, J. Liu, J. Wang, T. Zhao, L. Jiang and Y. Song, J. Phys. Chem. C, 2010, 114, 22303-22308.

20 B. Hatton, L. Mishchenko, S. Davis, K. H. Sandhage and J. Aizenberg, PNAS, 2010, 107, 10354-10359.

21 G. von Freymann, V. Kitaev, B. V. Lotsch and G. A. Ozin, Chem. Soc. Rev., 2013, 42, 2528-2554.

22 P. Jiang, G. N. Ostojic, R. Narat, D. M. Mittleman and V. L. Colvin, Adv. Mater., 2001, 13, 389-393.

23 B. Griesebock, M. Egen and R. Zentel, Chem. Mater., 2002, 14, 4023-4025.

24 H.-L. Li and F. Marlow, Chem. Mater., 2006, 18, 1803-1810.

25 P. Jiang and M. J. McFarland, J. Am. Chem. Soc., 2004, 126, 13778-13786.

26 A. Mihi, M. Ocaña and H. Míguez, Adv. Mater., 2006, 18, 2244-2249.

27 C. E. Finlayson, P. Spahn, D. R. Snoswell, G. Yates, A. Kontogeorgos, A. I. Haines, G. P. Hellmann and J. J. Baumberg, Adv. Mater., 2011, 23, 1540-15444.

28 A. Kontogeorgos, D. R. E. Snoswell, C. E. Finlayson, J. J. Baumberg, P. Spahn and G. P. Hellmann, Phys. Rev. Lett., 2010, 105, 233909.

29 H. S. Wong, M. Mackley, S. Butler, J. Baumberg, D. Snoswell, C. Finlayson and Q. Zhao, J. Rheol., 2014, 58, 397-409.

30 T. Ruhl and G. P. Hellmann, Macromol. Chem. Phys., 2001, 202, 3502-3505.

31 T. Ruhl, P. Spahn and G. P. Hellmann, Polymer, 2003, 44, 7625-7634.

32 O. L. J. Pursiainen, J. J. Baumberg, H. Winkler, B. Viel, P. Spahn and T. Ruhl, Adv. Mater., 2008, 20, 1484-1487.
33 C. G. Schäfer, B. Viel, G. P. Hellmann, M. Rehahn and M. Gallei, ACS Appl. Mater. Interfaces, 2013, 5, 10623-10632. 34 C. G. Schäfer, M. Gallei, J. T. Zahn, J. Engelhardt, G. P. Hellmann and M. Rehahn, Chem. Mater., 2013, 25, 2309-2318.

35 B. Pittenger, N. Erina and S. Chanmin, Bruker Application Note \#128, 2009.

36 B. V. Derjaguin, V. M. Muller and Y. P. Toporov, J. Colloid Interface Sci., 1975, 53, 314-326.

37 C. Graf and A. van Blaaderen, Langmuir, 2002, 18, 524-534.

38 C. G. Schäfer, S. Heidt, D. Scheid and M. Gallei, AAP Archives, 2014 , in press.

39 C. G. Schäfer, C. Lederle, K. Zentel, B. Stuhn and M. Gallei, Macromol. Rapid Commun., 2014, 35, 1852-1860.

40 W. Stöber, A. Fink and E. Bohn, J. Colloid Interface Sci., 1968, 26, 62-69.

41 N. Hatto, T. Cosgrove and M. Snowden, Polymer, 2000, 41, 7133-7137.

42 M. Karg, I. Pastoriza-Santos, L. M. Liz-Marzan and T. Hellweg, ChemPhysChem, 2006, 7, 2298-2301.

43 C. Liu, J. Guo, W. Yang, J. Hu, C. Wang and S. Fu, J. Mater. Chem., 2009, 19, 4764.

44 P. Jiang, K. Hwang, D. Mittleman, J. Bertone and V. Colvin, J. Am. Chem. Soc., 1999, 121, 11630-11637.

45 P. Jiang, J. F. Bertone and V. L. Colvin, Science, 2001, 291, 453-457.

46 H. Saito, Y. Takeoka and M. Watanabe, Chem. Commun., 2003, 2126.

47 Y. Takeoka and M. Watanabe, Adv. Mater., 2003, 15, 199-201. 48 Y. Takeoka and M. Watanabe, Langmuir, 2003, 19, 91049106.

49 C. G. Schäfer, D. A. Smolin, G. P. Hellmann and M. Gallei, Langmuir, 2013, 29, 11275-11283.

50 C. G. Schäfer, M. Biesalski, G. P. Hellmann, M. Rehahn and M. Gallei, J. Nanophotonics, 2013, 7, 070599.

51 C. G. Schäfer, S. Vowinkel, G. P. Hellmann, T. Herdt, C. Contiu, J. J. Schneider and M. Gallei, J. Mater. Chem. C, 2014, 2, 7960-7975.

52 D. Scheid, C. Lederle, S. Vowinkel, C. G. Schäfer, B. Stühn and M. Gallei, J. Mater. Chem. C, 2014, 2, 2583-2590.

53 O. L. J. Pursiainen, J. J. Baumberg, H. Winkler, B. Viel, P. Spahn and T. Ruhl, Opt. Express, 2007, 15, 9553-9561. 